Check for updates

Cite this: RSC Adv., 2019, 9, 10881

\title{
Effects of mycorrhizae and water conditions on perennial ryegrass growth in rare earth tailings
}

Received 20th December 2018 Accepted 27th March 2019

DOI: $10.1039 / c 8 r a 10442 e$

rsc.li/rsc-advances

\author{
Qiao Yang, (D) ${ }^{\text {a }}$ Zhongqiu Zhao, ${ }^{\text {ab }}$ Zhongke Bai, $^{\text {ab }}$ Hong Hou, $^{c}$ Ye Yuan, (D) ${ }^{a}$ \\ Anning Guo and Yufeng $\mathrm{Li}^{\mathrm{a}}$
}

Mycorrhizal symbioses, which include plant roots and arbuscular mycorrhizal fungi (AMF), can significantly enhance plant resistance and promote the absorption of soil nutrients by plants. A greenhouse experiment was conducted to investigate the effects of three AMF species (Glomus mosses, Glomus etunicatum and Glomus versiforme) on the height, biomass, malondialdehyde (MDA) and proline contents and antioxidant enzyme activities of perennial ryegrass (Lolium perenne) under different water supply treatments. Potted experimental soil samples were collected from the abandoned rare earth tailings in Ganzhou, Jiangxi. The results showed that all three AMF species infected ryegrass under the different treatments. Under severe drought stress, G. mosses had the most significant effects on the promotion of ryegrass performance. After inoculation, the height and whole-plant biomass of ryegrass increased by $60.44 \%$ and $150 \%$, respectively. In addition, inoculation with AMF significantly reduced the content of MDA and proline in the ryegrass leaves in all water supply treatments except the moderate drought stress treatment, in which there was no effect. The leaf antioxidant enzyme activity was also measured. The results showed that under severe drought stress, inoculation with Glomus mosses significantly increased the activities of CAT and SOD in ryegrass and enhanced the resistance of plants. A possible reason that AMF promotes host plant growth and enhances drought resistance is that AMF directly increases the absorption of soil water and minerals by host plant roots and indirectly improves the physiological metabolism of plants.

\section{Introduction}

According to current data, China is the country with the richest reserves of rare earth resources in the world, accounting for $50 \%$ of the total reserves worldwide. ${ }^{1}$ Twenty-two provinces in China have rare earth deposits, with most deposits being widely distributed and relatively concentrated in remote mountainous areas.

The ionic rare earth ore deposits are mostly distributed on the surface of exposed weathered granite with a thickness of approximately $9 \mathrm{~m}$ and were mainly mined in southern Jiangxi in the late 1970 s. $^{2}$ Due to irrational predatory mining, the backward mining technologies of mining enterprises and untimely environmental supervision, environmental protection problems in mining areas have become increasingly prominent during the 30 years of development and utilization of rare earth resources. According to the data, the use of the pool dipping process to produce $1 \mathrm{t}$ rare earth required stripping approximately $300 \mathrm{~m}^{2}$ of topsoil, which damaged the vegetation by approximately $150 \mathrm{~m}^{2}$, resulting in approximately $667 \mathrm{~m}^{2}$ of desertified land. The

${ }^{a}$ School of Land Science and Technology, China University of Geosciences, Beijing 100083, P. R. China. E-mail: zhongqiuzhao@163.com

${ }^{b}$ Key Laboratory of Land Consolidation and Rehabilitation Ministry of Land and Resources, Beijing 100035, China

${ }^{c}$ State Key Laboratory of Environmental Criteria and Risk Assessment, Chinese Research Academy of Environmental Sciences, Beijing 100012, China vegetation was seriously damaged during the mining process, resulting in almost no vegetation in the mining area. Vegetation damage caused the soil parent material to be directly exposed to the wind and sun for many years, resulting in poor soil and desertification. $^{3}$ The reduction in soil microbes and the destruction of the seed bank have caused the entire ecological structure to become unbalanced, making the natural ecological restoration of the abandoned mining areas more difficult. ${ }^{4}$ Most of the rare earth minerals are located in remote mountainous areas. The water in the tailing sand is mainly derived from precipitation, and its water retention capacity is poor. Drought is an important factor influencing the difficulty of vegetation restoration on rare earth tails. Therefore, it is essential to improve the ability of plants growing on rare earth tail sand to resist drought stress. The use of biotechnology has been a hot topic of research in terms of improving plant drought resistance.

Arbuscular mycorrhizae are reciprocal symbionts formed by Glomus fungi in the soil and more than $80 \%$ of higher plants. Studies have shown that mycorrhizal symbionts can promote the absorption and utilization of soil moisture and mineral nutrients by plant roots, protect host plants from drought stress, promote plant growth and increase plant viability in stressed habitats. ${ }^{5}$ They play an important role in facilitating ecosystem restoration and reconstruction. Inoculation with arbuscular mycorrhizal fungi (AMF) under water stress increases plant growth and yield. When 
variables such as habit, life cycle, and water conditions are controlled, the effects of inoculation of AMF on plants can still differ due to interaction effects. ${ }^{6}$ In a previous study, three types of AMF were inoculated under different environments experiencing water stress, which were characterized by poor soil nutrients, fragile ecological environments and rare vegetation. ${ }^{7}$ Inoculation with AMF in cold and arid areas can increase the survival rate of transplanted seedlings. ${ }^{8}$ Furthermore, inoculation with AMF under drought conditions can increase the biological yield of wheat, especially after the four-leaf stage. ${ }^{9}$ Under high concentrations of lead and cadmium, inoculation with AMF increases the yield of basil (Ocimum basilicum) and ensures its quality under heavy metal stress. ${ }^{10}$ Inoculation with AMF improves the rhizosphere soil in mining areas, the number of microorganisms is obviously improved, and a better mycorrhizal ecological effect is obtained. The presence of mycorrhizae promotes the stability of mining ecosystems and maintains their sustainability. ${ }^{\mathbf{1 1}}$ Under normal water supply and water control conditions, inoculation with Glomus etunicatum can increase the chlorophyll content of chickpea (Cicer arietinum) leaves, ${ }^{12}$ and inoculation with AMF enhances the activity of polyphenol oxidase and peroxidase (POD) while also reducing the activity of catalase (CAT).

Ryegrass has developed roots and produces clumps and many tillers and easily forms mycorrhizae under natural conditions. Plants inoculated with AMF can better adapt to arid environments. In recent years, AMF have been found to play an important role in the nutrition of plant-soil systems through joint action with plants, and they have great advantages that can be gradually applied to the ecological restoration and reconstruction of mining areas. However, there have been few studies on the use of AMF in rare earth mining restoration. There are also few reports on the phytoremediation of abandoned rare earth mines with microorganisms, and there has been no indepth study on the effects of phytoremediation.

Therefore, the application of AMF to improve the drought resistance of ryegrass has practical significance. ${ }^{\mathbf{1 3 , 1 4}}$ In this study, three types of AMF were inoculated under different water stress conditions characterized by poor soil nutrients, fragile ecological environments and rare vegetation to investigate the resistance of perennial ryegrass to water stress through the study of the effects on ryegrass growth and nutrient absorption. The objectives were to (1) screen the dominant strains that improve the resistance of ryegrass and (2) provide a scientific and technical basis for the study of AMF for rare earth tailing vegetation restoration.

\section{Materials and methods}

\subsection{Soil physicochemical properties}

The tailing soil used in the present study was collected from the abandoned rare earth mining area in southern Ganzhou $\left(24^{\circ} 38^{\prime}\right.$ $12.46^{\prime \prime} \mathrm{N}, 115^{\circ} 44^{\prime} 33.40^{\prime \prime}$ ), Jiangxi Province, China, where mining was discontinued more than 30 years ago. In the past, pool leaching and heap leaching were mainly used in rare earth mining areas. The damage to vegetation was substantial, resulting in serious soil erosion. ${ }^{15}$ Each pot was sampled at five-point to collect mixed soil samples. We also collected soil from unexploited mining areas as background soil. The heavy metals in the tailings heap were leached for a long time because of the heavy precipitation in this area. The mixed soil was naturally air dried and passed through a $2 \mathrm{~mm}$ mesh sieve. Several physiochemical properties of the soil and possible heavy metal contents were determined according to the Chinese standard methods given in (Table 1). The heavy metal content of the soil was determined from $0.50 \mathrm{~g}$ soil samples that were passed through a $0.20 \mathrm{~mm}$-sieve, followed by $\mathrm{HF}+\mathrm{HNO}_{3}+\mathrm{HClO}_{4}(3: 1: 1)$ digestion. Inductively coupled plasma mass spectrometry (ICP-MS) using an Agilent $7700 \times$ was then used to measure the heavy metal content. Determination of soil texture was used a laser particle size analyser. The soil $\mathrm{pH}$ was measured with electrode $\mathrm{pH}$ meter using a 1 : $2.5(\mathrm{w} / \mathrm{w})$ soil- $\mathrm{CaCl}_{2}$ water suspension (Denver Instrument UB-7 $\mathrm{pH} \mathrm{mV}{ }^{-1}$ Meter, ultraBASIC). The soil organic matter content was determined by a total organic carbon instrument (multi N/C 3100 TOC). The soil total nitrogen and phosphorus were determined by potassium dichromate-sulfuric acid digestion and the sulfuric acid-perchloric acid digestion method, respectively. The soil total potassium was determined by flame photometry method. The soil available nitrogen and phosphorus were measured by the $0.5 \mathrm{~mol} \mathrm{~L}^{-1}$ sodium bicarbonate and alkali hydrolysis diffusion methods, respectively. The soil available potassium were measured by ammonium acetate-flame photometer method. ${ }^{16}$ All soils were sterilized with dry heat at $170{ }^{\circ} \mathrm{C}$ for 6 hours.

\subsection{Experimental design}

The collected soil was sterilized by a series of autoclaving steps (170 ${ }^{\circ} \mathrm{C}$ for 6 hours, 1 day intervals between each step). A pot experiment was conducted in a semi-enclosed greenhouse under a normal light/dark cycle at the China Academy of Environmental Sciences in Beijing, China, between May and April 2017. The results of the soil physical and chemical properties analysis showed that the content of heavy metals in the soil did not exceed the relevant secondary standards in the Environmental Quality Standard for Soils of China, GB15618-1995 (Table 1). The soil was sandy loam (82.94\% sand, $2.17 \%$ clay, and $14.89 \%$ silt). Each pot was filled with $20 \mathrm{~g}$ AMF and $1 \mathrm{~kg}$ soil that was irradiated and sterilized after passing through a $2 \mathrm{~mm}$ sieve. In this study, four experimental treatments were used, each of which included three field soil water contents, W1, W2, W3, representing 60\% (normal water supply), $40 \%$ (moderate stress) and $20 \%$ (severe stress) of the field water content, respectively. Each treatment was repeated 3 times in random order. The three AMF used in this study were Glomus mosses (G.m), Glomus etunicatum (G.e) and Glomus versiforme (G.v). A control treatment (CK) was established without the addition of AMF. The microbial inoculum was provided by the Institute of Plant Nutrition and Resources, Beijing Academy of Agriculture and Forestry Sciences. The inoculum was obtained through sorghum propagation and contained mycorrhizal fungal spores, host plant root segments and mycelia in the plant rhizosphere containing mixed soil. The seeds of ryegrass were disinfected with $10 \% \mathrm{H}_{2} \mathrm{O}_{2}$ for 10 minutes before sowing. After washing with sterile water, the seeds were evenly placed in 100 mm-diameter Petri dishes with a double layer of filter paper. The seeds were sown when they appeared on the surface of the filter 


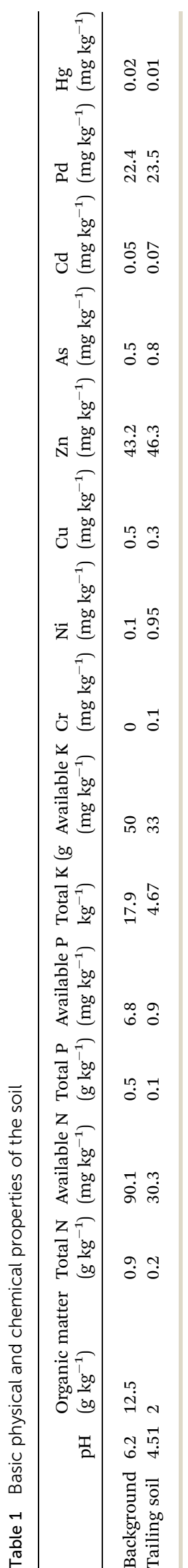

paper at a temperature of $20-25{ }^{\circ} \mathrm{C}$. Thirty ryegrass seeds were sown in each pot, and the plants were randomly arranged. After 30 days, 10 seedlings with a uniform growth status were kept, and the rest were discarded. The drought stress experiment was carried out when the ryegrass had grown for 30 days. Three soil relative water content treatments were established, which were $60 \%, 40 \%$, and $20 \%$ of the field water contents. Water was added according to the remaining moisture, which was determined by weighing the pots at least once a day starting on the thirty-first day of the experiment. When the weather was hot, the pots were weighed in both the morning and evening. In addition, the temperature and humidity of the greenhouse were maintained at $18-25^{\circ} \mathrm{C}$ and $65 \%$ to $80 \%$, respectively. Natural lighting, shading and air humidification were used to reduce transpiration. The overall aim of these conditions was to maintain the soil moisture content in the pots.

\subsection{Plant analysis and determination methods}

After 50 days, samples of the ryegrass roots were taken to determine the infection rate. Ryegrass leaves were collected to determine the plant height, plant biomass, malondialdehyde (MDA) content and proline and oxidase activity.

2.3.1 Analysis of AMF infection. At the end of the whole growth experiment, the roots of the plants were dug out of the soil and carefully cleaned with deionized water until the soil was removed, and they were weighed separately from the aboveground biomass. The fresh roots were preserved in a fixed solution of formaldehyde, glacial acetic acid, and ethanol with a volume ratio of $12.5: 12.5: 200$ in the refrigerator at $4{ }^{\circ} \mathrm{C}$ for analysis. The AMF infection rate was measured by the Phillips and Hayman method. ${ }^{\mathbf{1 7}}$ The determination of the infection rate is a simple and direct way to compare the number of AMF in the soil and culture. The usual determination steps include fixation, transparency, staining, production, observation and estimation. Fixation: the roots were washed with deionized water, cut into $1 \mathrm{~cm}$-long segments and placed in a $50 \mathrm{~mL}$ polyethylene bottle. Some roots were randomly selected and immersed in formaldehyde, glacial acetic acid and 50\% ethanol (v:v:v= $13: 5: 200)$ for more than 4 hours. Transparency: approximately $2 \mathrm{~g}$ root segments were rinsed with deionized water in a $50 \mathrm{~mL}$ beaker, immersed in a $10 \% \mathrm{KOH}$ solution, and boiled for 10 minutes on an electric heating plate. After heating, the roots were rinsed several times with deionized water until the water was colourless. Staining: the solution in the beaker containing transparent root segments was stained with glacial acetic acid and ink ( $\mathrm{v}: \mathrm{v}=19: 1)$ and boiled for approximately 5 minutes. The excess dye was poured out, and then the roots, which had been dyed many times were washed with acetated deionized water and stored in deionized water. Production: tweezers were used to place the stained root segments on clean glass slides. Five slides were produced for each sample, with 8 root segments on each slide, for a total of 40 root segments. Then, 2-3 drops of lactic acid were added to the samples, which were then covered with cover glass, ensuring that there were no bubbles, and then observed under an optical microscope.

2.3.2 Physiological and biochemical analysis of plants. The height of the plants was measured with a ruler. The collected 
plant samples were washed with deionized water and then divided into aboveground and root biomass with ceramic scissors. Each part of the sample was placed in an oven and dried at $65{ }^{\circ} \mathrm{C}$ for 72 hours, and the dry matter of the samples was weighed. The proline content in the plants was measured using the three-ketone colorimetry method, ${ }^{18}$ and the MDA content in the plants was measured by the thiobarbituric acid colorimetry method. ${ }^{19}$ Three grams of leaves were cut into pieces and added to $30 \mathrm{~mL}$ of phosphoric acid buffer. The leaves were ground into a homogenate, centrifuged, extracted, and combined with the supernatant to a fixed volume, and then the enzyme solution was prepared for refrigeration. Superoxide dismutase (SOD) activity was determined by the nitrogen blue four azole (NBT) method..$^{20}$ A sample of $0.5 \mathrm{~mL}$ of the above enzymatic liquor was collected, and then $1.5 \mathrm{~mL}$ of $0.05 \mathrm{~mol} \mathrm{~L}^{-1}$ phosphate buffer, $0.3 \mathrm{~mL}$ of $130 \mathrm{mmol} \mathrm{L}^{-1}$ Met solution, $0.3 \mathrm{~mL}$ of $0.75 \mathrm{mmol} \mathrm{L}^{-1}$ NBT solution, $0.3 \mathrm{~mL}$ of $0.1 \mathrm{mmol} \mathrm{L}^{-1}$ EDTA-Na $_{2}$ solution and $0.3 \mathrm{~mL}$ of $0.02 \mathrm{mmol} \mathrm{L}^{-1}$ riboflavin were added. Using a dark tube as a blank control, UV absorbance was measured at $560 \mathrm{~nm}$. The activity of CAT was determined by ultraviolet absorption. ${ }^{21}$ A sample of the enzyme in the amount of $2.5 \mathrm{~mL}$ was added to $2.5 \mathrm{~mL}$ of $0.1 \mathrm{~mol} \mathrm{~L}^{-1} \mathrm{H}_{2} \mathrm{O}_{2}$. Afterwards, $2.5 \mathrm{~mL}$ $10 \% \mathrm{H}_{2} \mathrm{SO}_{4}$ was added to the sample, which was then heated in a $30{ }^{\circ} \mathrm{C}$ water bath for 10 minutes, and then this solution was titrated with $0.1 \mathrm{~mol} \mathrm{~L}^{-1} \mathrm{KMnO}_{4}$ until the pink colouration disappeared. The UV absorbance was measured at a wavelength of $240 \mathrm{~nm}$. The activity of POD was determined by the guaiacol method. ${ }^{22}$ A sample of the above enzyme solution in the amount of $0.1 \mathrm{~mL}$ was taken, and then $2.9 \mathrm{~mL}$ of $0.05 \mathrm{~mol} \mathrm{~L}^{-1}$ phosphate buffer, $1.0 \mathrm{~mL}$ of $2 \% \mathrm{H}_{2} \mathrm{O}_{2}$, and $1.0 \mathrm{~mL}$ of $0.05 \mathrm{~mol} \mathrm{~L}^{-1}$ guaiacol was added. The solution was heated in a $37{ }^{\circ} \mathrm{C}$ water bath for 15 minutes, and then $2.0 \mathrm{~mL}$ of $20 \%$ trichloroacetic acid was added to terminate the reaction. The solution was then centrifuged, and the absorbance was measured at a wavelength of $470 \mathrm{~nm}$.

\subsection{Statistical analysis}

The mycorrhizal infection rate was calculated as follows:

the mycorrhizal infection rate $=\frac{\text { number of roots }}{\text { total number of roots }} \times 100 \%$

All the data obtained in the experiment were plotted with Origin8.5. The programs of excel 2010 and SPSS 16.0 were used to analyse the statistical variance of the data. Duncan's new complex range analysis was used to analyse the data under different water supply treatments, and one-way ANOVA was performed to analyse the data under the same water condition.

\section{Results}

\subsection{Rate of AMF infection}

The rate of AMF infection was determined on the basis of the microstructure of the infected ryegrass roots by identifying the spores, external hyphae and internal hyphae (Fig. 1). From this information, it can be seen that the roots were infected by AMF. Under the AMF and water stress treatments, there were mycorrhizal infecting structures in the ryegrass root system (Table 2), and no
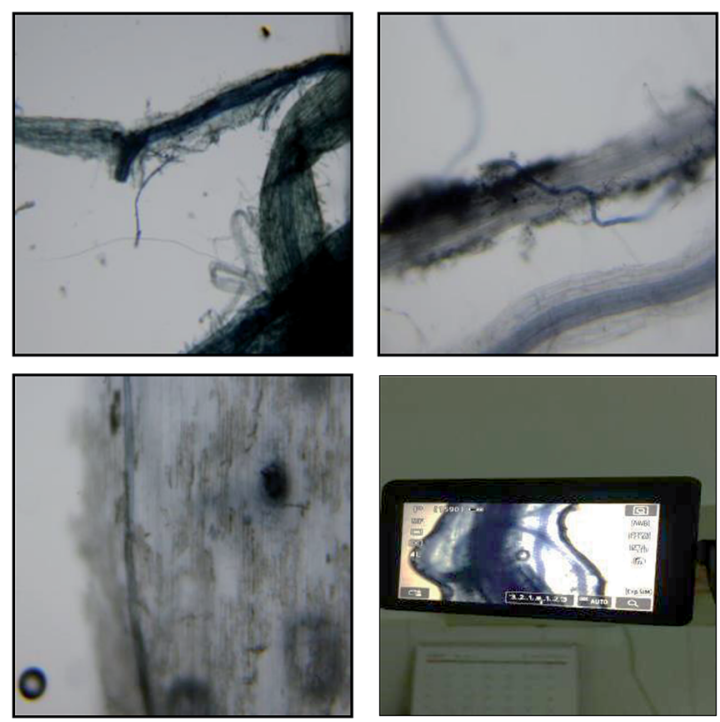

Fig. 1 Microstructure of the infected ryegrass roots $(100 \times)$.

mycorrhizal structures were found in the CK treatment, in which AMF inoculation did not occur. The mycorrhizal infection rate decreased significantly from $76.44 \%$ to $51.06 \%$ in the treatment of G.m inoculation from the sufficient water supply to severe drought conditions. It is concluded that the ability of G.m to infect ryegrass roots is greater than that of G.e and even greater than that of G.v under the same water supply treatment conditions. Moderate and severe drought stress significantly reduced the mycorrhizal infection rate of the three AMF species. With an increase in drought stress, the mycorrhizal dependence rate of ryegrass increased with AMF inoculation, and there was a significant interaction between the water supply and AMF treatments $(P<0.05)$.

\subsection{Effects of AMF and water stress on the growth of ryegrass}

The plant height and biomass of ryegrass changed under the different AMF and water supply treatments. Inoculation with AMF increased the plant height and biomass of ryegrass under the same moisture treatment (Fig. 2). However, ryegrass growth in the severe water supply treatment was better than that in the severe drought treatment, and the effect of AMF inoculation on the growth of ryegrass was significant under severe drought stress. The average whole plant biomass was $1.5 \mathrm{~g}$ when inoculated with G.m, which was 2.5 times higher than that in the CK treatment. In comparison to the growth of ryegrass when it was inoculated with G.e and G.v, the growth was better promoted by inoculation with G.m, with the plant height and biomass increasing by $60.44 \%$ and $150 \%$, respectively, after inoculation. The root-shoot ratio was also affected by the different AMF and water supply treatments. The root-shoot ratio increased with increasing drought stress. After treatment with G.m, the root-shoot ratio increased with increasing drought stress, while the results from the G.e. treatment were reversed, and the root-shoot ratio following G.v inoculation was basically unchanged. Under the same water supply conditions, the mycorrhizal infection rate of G.m was higher than that of G.e. Therefore, the root ratio of ryegrass treated with G.m was higher than that when treated with G.e. 
Table 2 Effects of AMF on the growth of host plants under water stress ${ }^{a}$

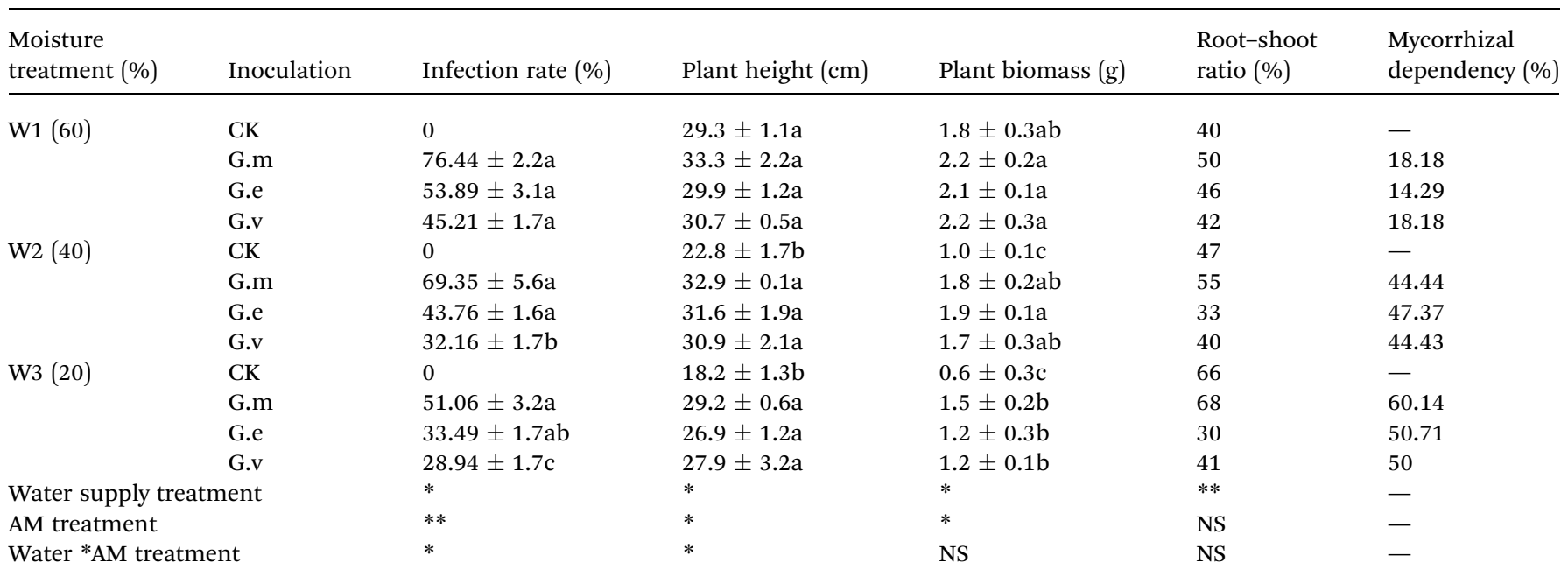

${ }^{a}$ Different small letters in the same column indicate significant differences at the 0.05 level among treatments. * Indicates that the treatment is extremely significantly different from the control $(P<0.05)$. ${ }^{* *}$ Indicates that the treatment is significantly different from the control $(P<0.01)$.

\subsection{Effects of AMF and water stress on the content of MDA and proline in ryegrass}

In the absence of inoculation, the MDA content in ryegrass leaves increased with increasing water stress (Fig. 2a). Under severe drought stress, the MDA content of ryegrass leaves
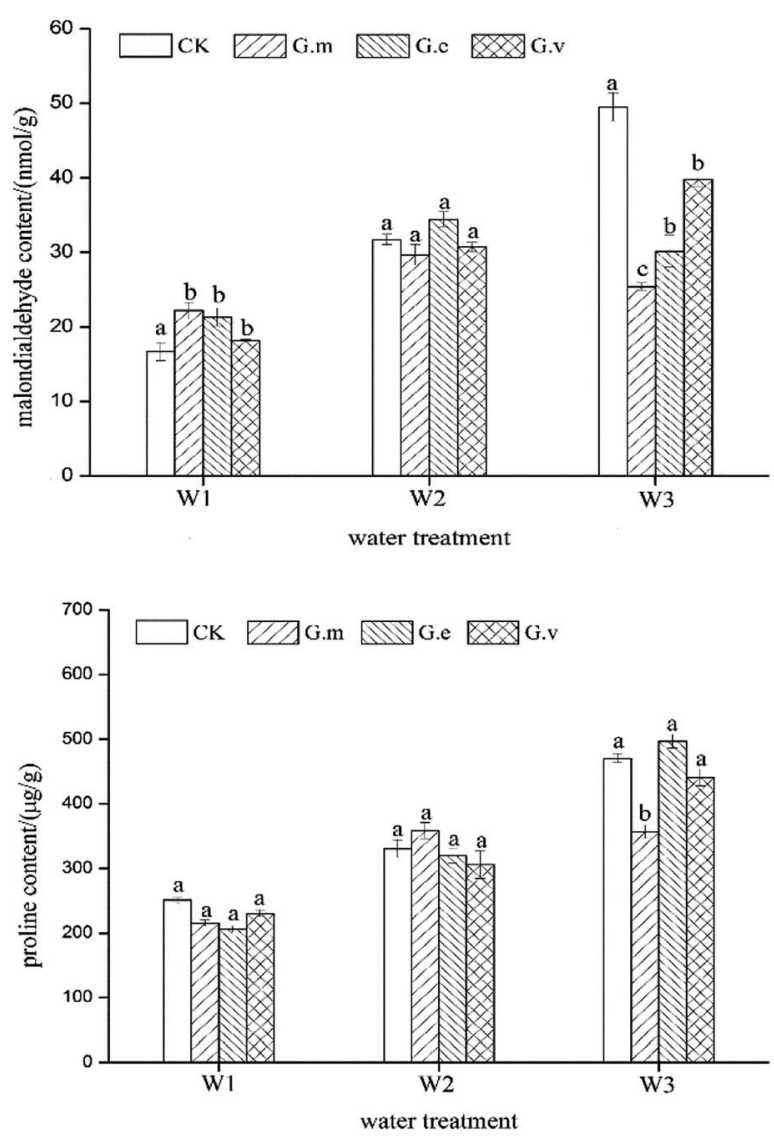

Fig. 2 MDA and proline contents of ryegrass under three different water supply treatments. inoculated with the three types of AMF was significantly lower than that of ryegrass in the non-inoculated treatment $(P<0.05)$, indicating that the damage to the cell membrane caused by drought stress was lower than that in the non-inoculation treatment. Under this water supply treatment, the most inactivated MDA content occurred in the ryegrass inoculated with G.m, followed by that inoculate with G.e and G.v. Under moderate drought stress, there were no significant differences among inoculation with the three types of AMF and the noninoculation treatment. Under the normal water supply conditions, inoculation with the three types of AMF significantly increased the content of MDA in ryegrass leaves in comparison with the non-inoculation treatment. The results of this experiment are consistent with the results of $\mathrm{Zhu}^{23}$

Under normal water supply conditions, inoculation with the three types of AMF reduced the content of proline in the ryegrass, but the difference was not significant $(P>0.05)$ (Fig. 2b). There were no significant differences in the proline content of ryegrass among the AMF inoculation and noninoculated treatments under moderate water stress conditions. Under severe drought stress, the content of proline in the ryegrass increased significantly in association with AMF inoculation, indicating that ryegrass initiated osmotic adjustment to prevent drought stress. Inoculation with both G.m and G.v reduced the proline content of the ryegrass. The G.m treatment reduced the proline content by $24.24 \%$, while the inoculation of

b G.e increased the proline content by $4.41 \%$. This indicates that the G.m, G.v and G.e treatments have different regulatory effects on ryegrass under drought stress.

\subsection{Effects of AMF and water stress on protective enzymes in ryegrass leaves}

The level of CAT in the leaves reflects the ability of oxygen free radicals to damage the leaves. In the three water supply treatments, the CAT activity of the leaves of ryegrass inoculated with 
AMF was higher than that in the non-inoculated treatment (Fig. 3a). The CAT activity of leaves in the treatment without inoculation with AMF gradually increased with increased drought stress, reaching a maximum of $102.39 \mathrm{U} \mathrm{g}^{-1} \mathrm{~min}^{-1}$ under severe drought stress. The CAT activity in plants inoculated with G.m, G.e, and G.v increased by more than $36.20 \%$, $10.21 \%$, and $19.63 \%$, respectively. The CAT activity in the plants inoculated with G.m was $23.59 \%$ and $13.85 \%$ higher than that of plants inoculated with G.e and G.v, respectively. One-way ANOVA showed that there were significant differences $(P<$ 0.05) between the G.m and CK treatments and between the G.e, and G.v treatments under moderate and severe drought stress.

The activity of POD can reflect the metabolic status of plants and their adaptability to the external environment, and the POD activity of plants that were not inoculated with AMF increased first
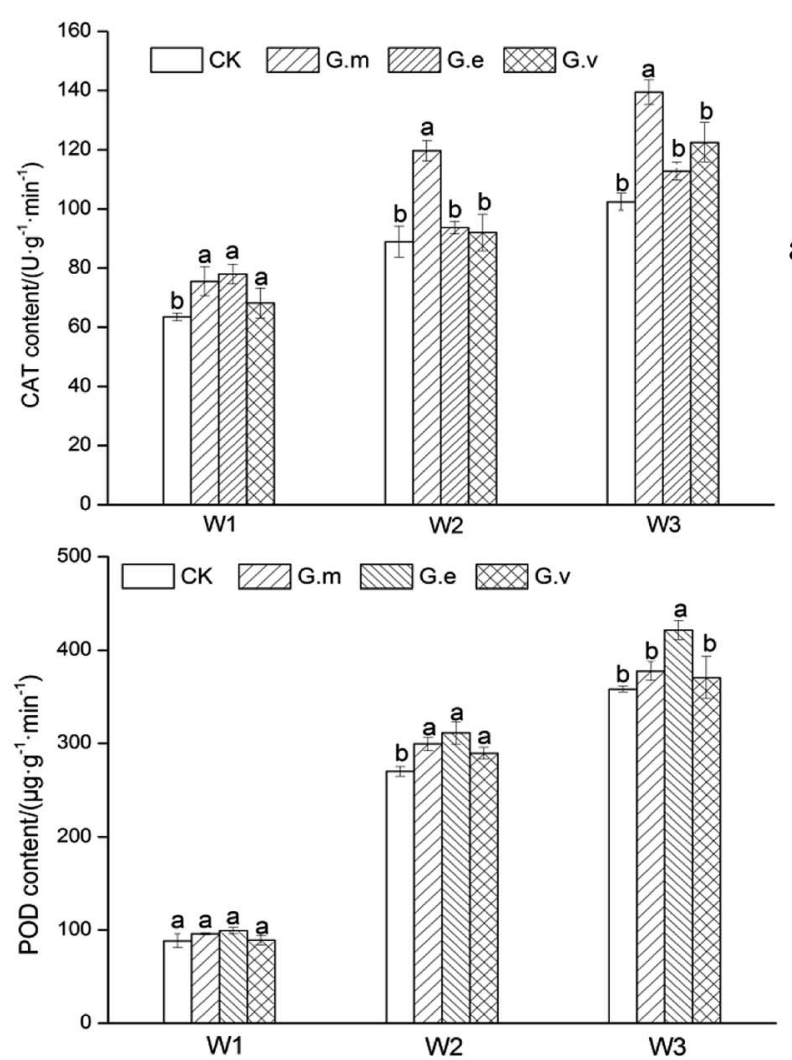

b

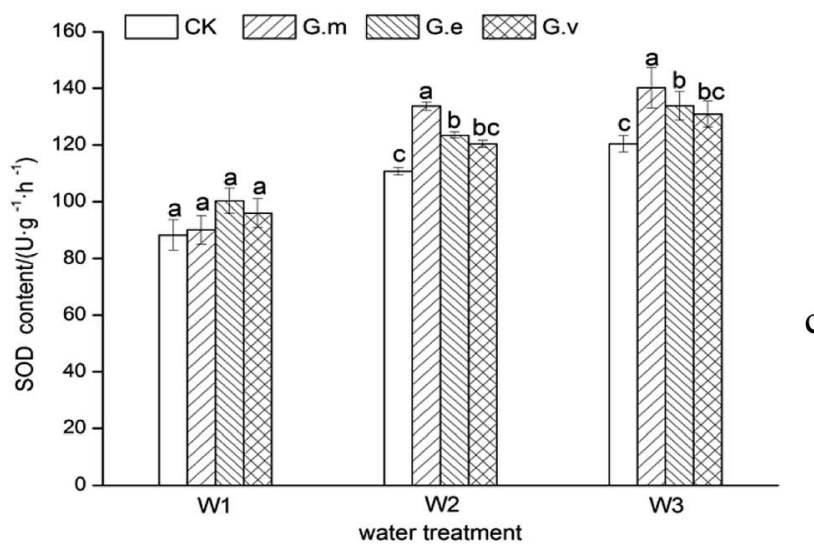

Fig. 3 Superoxide dismutase activity of ryegrass under three different water supply treatments. and then decreased with an increase in drought stress (Fig. 3b). Among all three water supply treatments, the POD activity of ryegrass leaves inoculated with AMF was higher than that of noninoculated leaves. Under severe drought stress, the POD activity of ryegrass leaves inoculated with G.e was the highest, at $401.73 \mathrm{U}$ $\mathrm{g}^{-1} \mathrm{~min}^{-1}$. The POD activity in the plants inoculated with G.m, G.e and G.v in this treatment was $47.03 \%, 65.20 \%$ and $52.42 \%$ higher than that in the non-inoculated plants. The POD activity in the G.e and CK treatments was significantly different $(P<0.05)$ under severe drought stress, but the POD activity was not significantly different in these treatments in the normal water supply and moderate drought treatments.

Reactive oxygen species and free radicals increase under adverse conditions, and SOD catalyses the disproportionation reaction to generate hydrogen peroxide and oxygen from reactive oxygen species, thereby protecting cells from them. ${ }^{24}$ As drought increased, the activity of ryegrass SOD also increased (Fig. 3c); the SOD activity of ryegrass leaves was $88.32 \mathrm{U} \mathrm{g}^{-1} \mathrm{~h}^{-1}$ under the normal water supply treatment and increased to 130.39 $\mathrm{U} \mathrm{g}^{-1} \mathrm{~h}^{-1}$ under severe drought stress. The SOD activity in the ryegrass leaves inoculated with the three types of AMF was higher than that in the control. The order of SOD activity in the leaves was G.m > G.e > G.v. Under the treatments of moderate and severe drought stress, the SOD activity under inoculation with G.m was significantly different from that in the control $(P<0.05)$, but the difference was not significant under the normal water supply treatment.

\section{Discussion}

Daviess's studies have shown that the infection rates of ryegrass decline with an increase in drought stress. ${ }^{25}$ The infection rate results in this study are consistent with this trend: from a normal water supply to severe drought stress, the rates of ryegrass infection by the three AMF were significantly reduced, and the biomass of ryegrass also declined. Inoculation with AMF also significantly promoted the growth of the aerial parts and roots of amorpha (Amorpha fruticosa Linn). Inoculation with AMF helps to establish a mutually beneficial symbiotic relationship between mycorrhizal fungi and plants. ${ }^{15}$ Staddon's research indicates that water stress can affect infection by the three AMF species used in this study by stimulating the germination of AMF and endophytic fungal spores and by affecting the growth and extension of hyphae. ${ }^{26}$ There was a significant interaction between the water supply and AMF treatments in this study $(P<0.05)$. This interaction was mainly due to the different activities and infestation advantages of the three AMF species under different water conditions. The ryegrass infection rates of the three types of AMF differed under the same water supply conditions, indicating that the symbiotic relationship between AMF and ryegrass is closely related to the species of AMF. Fajardo's research shows that plants and mycorrhizae coexist in a selective relationship, such as that between alfalfa and native strains of AMF. ${ }^{27}$ In the current experiment, G.m had the most significant effect on the promotion of ryegrass growth, indicating that G.m has a higher affinity to ryegrass and can play a better role in the stress environment. 
The symbiotic relationship between AMF and plants will have different effects on the nutrient absorption and the physiological and biochemical reactions of host plants, thereby increasing the tolerance of host plants to various environmental stressors. ${ }^{28}$ In addition, the distribution of host plant biomass is affected by water stress and AMF. This study showed that the different water supply treatments significantly affected the root-shoot ratio of ryegrass, while the effects of the AMF treatments were not significant. This result is similar to those of a study by Al-Karaki, who showed that inoculation with AMF had no significant effect on the root-shoot ratio of wheat of different genotypes. ${ }^{29}$ When AMF is used for reclaiming in mining areas, it not only improves the soil rhizosphere enzyme activity in the mining area, but also improves the soil rhizosphere micro-ecological environment. ${ }^{30}$ The mycorrhizal symbiosis formed by AMF and roots promotes the absorption of nutrients by roots and the growth of parts of the ryegrass plant and also improves the growth of underground parts, explaining why there was no significant effect on the rootshoot ratio. The results showed that G.m formed a good symbiosis with ryegrass roots and had a high mycorrhizal infection rate; therefore, more photosynthetic products are transported to the roots, indicating that there is sufficient carbon available for the formation of the mycorrhizal symbiosis. In contrast, the G.e treatment had a different effect on the root-shoot ratio and growth of the host plant.

The results showed that under severe drought stress, inoculation with AMF significantly reduced the content of MDA in the leaves of the host plants, indicating that AMF can reduce damage to the cell membrane system. ${ }^{31} \mathrm{MDA}$ is a peroxide of cell membrane lipids, and plant tissues are subjected to oxidative stress under adverse conditions. When drought occurs, the plant cell membrane system is damaged, resulting in a significant increase in the amount of MDA in plants and death in plant cells. ${ }^{32}$ The results of this study showed that inoculation with the three AMF significantly reduced the MDA content in leaves under severe drought stress. The difference between the G.m treatment and the G.e and G.v treatments was significant, indicating that lipid peroxidation was relatively low and that strong drought resistance occurred. In agreement with Qiangsheng $\mathrm{Wu}$ and other results of research on seedlings, inoculation of plants with AMF can effectively alleviate the degree of drought stress and indirectly improve the water metabolism of plants. ${ }^{33}$ The accumulation of osmotic adjustment substances, such as proline, is one of the basic adaptations of plants to drought stress. The accumulation of these substances can regulate the intracellular osmotic potential, maintain the water balance, and protect the enzyme activities required for important metabolic activities in the cells, and it is generally considered that drought-tolerant varieties have stronger osmotic adjustment ability. ${ }^{34}$ The results of this study showed that the proline content of ryegrass gradually increased in the inoculation treatments as the drought stress increased. Under severe drought stress, inoculation with G.m and G.v reduced the proline content compared to that in the noninoculation treatment, and this difference was significant for G.m. However, inoculation with G.e increased the proline content of leaves, indicating that the symbiotic mycorrhizal system formed by G.m and ryegrass is more sensitive to drought and that G.m can facilitate a more rapid metabolic response, resulting in stronger drought resistance. Many studies have shown that AMF inoculation can improve the yield of corn, wheat, soybean and other major food crops, improve the nutritional status of crops, and enhance the resistance of crops to drought, salinity and other adverse environmental stresses. ${ }^{35}$ At the same time, it also showed that different AMF undergo different osmotic adjustments when associated with ryegrass, which may be related to the influence of the different AMF on host plant biomass allocation.

The growth and metabolism of plants are in a stable state under a normal water supply. Plant cells produce a large number of reactive oxygen and superoxide radicals due to metabolic obstruction when suffering from drought stress. These reactive oxygen species and superoxide radicals peroxidize cell membrane lipids with their strong oxidative properties, leading to membrane system and organelle damage and metabolic activity disorders. ${ }^{36}$ Plants will actively regulate the rate of reactive oxygen species production in the cells through a protective system, thereby reducing the damage caused by cell membrane lipid peroxidation.

Protection systems are divided into enzymatic defence systems and non-enzymatic defence systems. Higher activities of SOD, POD and CAT enzymes in the enzymatic defence system result in a stronger ability to eliminate oxygen free radicals and therefore stronger drought resistance in plants. Mycorrhizal plants are more adaptable under adverse conditions such as drought and saline.The activity of antioxidant enzymes such as superoxide dismutase (SOD), catalase (CAT) and peroxidase (POD) in the body is significantly improved, which can slow down the oxidative damage of plants and enhance drought resistance. ${ }^{37,38}$ The results of this study showed that the inoculated AMF mainly increased the enzyme activities (SOD, POD, CAT) to eliminate the accumulation of reactive oxygen species caused by drought stress, thereby reducing the damage caused by cell membrane lipid peroxidation. Studies have shown that inoculation can increase the CAT activity of Caragana korshinskii Kom. Leaves. ${ }^{39}$ Amiri found that two AMF (Glomus intraradices and Glomus mosseae) significantly increased the CAT activity of Pelargonium graveolens L'Herit. under drought stress. ${ }^{40}$

Inoculation with G.m significantly increased the contents of CAT and SOD in ryegrass under severe drought stress, while inoculation with G.e significantly increased the POD content, indicating that the three microbial agents differ in terms of eliminating the regulation of reactive oxygen species in the leaves of ryegrass under drought stress. Research suggests that POD activity is higher in the late stages of stress and that inoculation with AMF can significantly increase POD activity in the early stages of plant growth and enhance the ability of plants to cope with environmental stress in the middle and late growth stages. ${ }^{41}$ Water shortage can cause cell membrane damage, and POD activity immediately increases to a critical level to remove peroxide free radicals in plants. Inoculation with AMF can greatly increase this critical level. This is consistent with the report that existing inoculated fungi can significantly increase plant POD activity. ${ }^{42}$ 


\section{Conclusions}

(1) Ryegrass has unique relationships with the three tested AMF. Under all water supply treatments, G. mosses infected ryegrass at the highest rate. The height and biomass of the inoculated plants significantly increased under drought stress, while inoculation with AMF promoted the growth index of ryegrass under severe drought stress.

(2) The MDA and proline contents in the ryegrass leaves gradually increased with greater water stress when the plants were not inoculated. Under severe drought stress, the MDA and proline contents in the leaves of ryegrass treated with G.m were significantly lower than those from plants that were not inoculated.

(3) Under moderate and severe drought stress, inoculation with AMF significantly increased the antioxidant enzyme activity of ryegrass leaves and effectively improved the stress resistance of rare earth tailing plants. Inoculation with G.m strains had the most significant effect on drought resistance.

\section{Conflicts of interest}

There are no conflicts to declare.

\section{Acknowledgements}

This study was supported by the Foundation for Public Welfare of the Ministry of Land and Resources of China (No. 2015110822). We also thank Prof. Zhao Zhongqiu for the guidance during the writing and modifying manuscript.

\section{Notes and references}

1 Z. G. Zhong, H. P. Zhou and J. Hu, Met. Mine, 2017, 12, 76-81. 2 S. Ling, X. Wu and Y. Ren, Chem. Erde, 2015, 75, 403-417.

3 Y. Pan and H. Li, Environ. Manage., 2016, 57, 879-893.

4 S. E. Smith and D. J. Read, Mycorrhizal Symbiosis, 2nd edn, 1997.

5 Z. Y. Chen, X. Z. Cao and A. P. Peng, J. Agro-Environ. Sci., 2012, 31, 2101-2107.

6 A. A. R. Kula and D. C. Hartnett, Plant Ecol., 2015, 216, 1-9.

7 B. Jayne and M. Quigley, Mycorrhiza, 2014, 24, 109-119.

8 B. E. Davidson, S. J. Novak and M. D. Serpe, Mycorrhiza, 2016, 26, 1-14.

9 G. Al-Karaki, B. Mcmichael and J. Zak, Mycorrhiza, 2004, 14, 263-269.

10 A. Prasad, S. Kumar and A. Khaliq, Biol. Fertil. Soils, 2011, 47, 853-861.

11 S. P. Li, Y. L. Bi, W. P. Kong, J. Wang and H. Y. Yu, Environmental Sciences, 2013, 34, 4455-4459.

12 Y. Sohrabi, W. Weisany and K. Ghasemi-Golezani, Russ. J. Plant Physiol., 2012, 59, 708-716.

13 J. N. Klironomos, J. Mccune and M. Hart, Ecol. Lett., 2010, 3, 137-141.
14 M. Ruiz Sánchez, R. Aroc and Y. Muñoz, J. Plant Physiol., 2010, 167, 862-869.

15 Y. X. Li, L. Zhang and X. M. Zhou, Chin. Rare Earths, 2010, 31, 80-85.

16 S. D. Bao, Soil agrochemical analysis, China Agric. Press, 2000.

17 J. M. Phillips and D. S. Hayman, Trans. Br. Mycol. Soc., 1970, $55,158$.

18 P. Lei, Z. Xu and J. Liang, Plant Growth Regul., 2016, 78, 1-9. 19 S. Malar, S. S. Vikram and P. J. Favas, Bot. Stud., 2016, 55, 111.

20 J. Fukami, C. D. L. Osa and F. J. Ollero, Funct. Plant Biol., 2017, 45, 328-339.

21 S. Azarabadi, H. Abdollahi and M. Torabi, Eur. J. Plant Pathol., 2017, 147, 279-294.

22 W. Fang and C. H. Kao, Plant Sci., 2000, 158, 71-76.

23 Y. Zhu, J. L. Xiong and G. C. Lu, Acta Ecol. Sin., 2015, 35, 2419-2427.

24 Y. Chen, P. H. Chan and R. A. Swanson, Glia, 2015, 33, 343347.

25 F. T. Davies Jr, V. Olalde-Portugal and L. Aguilera-Gomez, Sci. Hortic., 2002, 92, 347-359.

26 P. L. Staddon, R. Gregersen and I. Jakobsen, Glob. Change Biol., 2004, 10, 1909-1921.

27 B. E. Roldan-Fajardo, New Phytol., 2010, 127, 115-121.

28 B. Jayne and M. Quigley, Mycorrhiza, 2014, 24, 109-119.

29 G. Al-Karaki, B. Mcmichael and J. Zak, Mycorrhiza, 2004, 14, 263-269.

30 Y. X. Zhang, Y. L. Bi and Y. X. Zhang, North. Hortic., 2014, 38, 161-164.

31 Y. Sohrabi, G. Heidari and W. Weisany, Symbiosis, 2012, 56, 5-18.

32 F. Zhao, L. Wang and H. Gao, Environ. Pollut. Control J., 2012, 34, 40-44.

33 Q. S. Wu and R. X. Xia, J. Plant Physiol. Mol. Biol., 2004, 30, 583-588.

34 Z. J. Liu, X. L. Zhang and J. G. Bai, Sci. Hortic., 2009, 121, 138143.

35 G. Selvakumar, C. C. Shagol and K. Kim, BMC Plant Biol., 2018, 18, 109.

36 M. Marmiroli, F. Pietrini and E. Maestri, Tree Physiol., 2011, 31, 1319-1334.

37 H. Ahmad, S. Hayat and M. Ali, Ecol. Evol., 2018, 8, 57245740.

38 E. Domokos, L. Jakab-Farkas and B. Darko, Front. Plant Sci, 2018, 9, 478.

39 X. He, T. Liu, X. An and L. Zhao, Acta Ecol. Sin., 2009, 29, 4752.

40 R. Amiri, A. Nikbakht and N. Etemadi, Sci. Hortic., 2015, 197, 373-380.

41 M. Dacosta and B. Huang, J. Am. Soc. Hortic. Sci., 2007, 132, 319-326.

42 J. P. Ball, G. M. Barker and R. A. Prestidge, J. Chem. Ecol., 1997, 23, 1419-1434. 